\title{
Synthesis, characterization and gas sensing property of hydroxyapatite ceramic
}

\author{
M P MAHABOLE, R C AIYER ${ }^{\dagger}$, C V RAMAKRISHNA, B SREEDHAR ${ }^{\ddagger}$, \\ and $R$ S KHAIRNAR* \\ School of Physical Sciences, Swami Ramanand Teerth Marathwada University, Nanded 431 606, India \\ 'Department of Physics, University of Pune, Pune 411 007, India \\ ${ }^{\ddagger}$ Indian Institute of Chemical Technology, Hyderabad 500 007, India
}

MS received 16 November 2004; revised 9 August 2005

\begin{abstract}
Hydroxyapatite (HAp) biomaterial ceramic was synthesized by three different processing routes viz. wet chemical process, microwave irradiation process, and hydrothermal technique. The synthesized ceramic powders were characterized by SEM, XRD, FTIR and XPS techniques. The dielectric measurements were carried out as a function of frequency at room temperature and the preliminary study on CO gas sensing property of hydroxyapatite was investigated. The XRD pattern of the hydroxyapatite biomaterial revealed that hydroxyapatite ceramic has hexagonal structure. The average crystallite size was found to be in the range 31-54 nm. Absorption bands corresponding to phosphate and hydroxyl functional groups, which are characteristic of hydroxyapatite, were confirmed by FTIR. The dielectric constant was found to vary in the range 913 at room temperature. Hydroxyapatite can be used as $\mathrm{CO}$ gas sensor at an optimum temperature near $125^{\circ} \mathrm{C}$. X-ray photoelectron spectroscopic studies showed the $\mathrm{Ca} / \mathrm{P}$ ratio of 1.63 for the HAp sample prepared by chemical process. The microwave irradiation technique yielded calcium rich HAp whereas calcium deficient HAp was obtained by hydrothermal method.
\end{abstract}

Keywords. Hydroxyapatite; biomaterial; ceramics; gas sensor; X-ray photoelectron spectroscopy; infrared spectroscopy.

\section{Introduction}

Hydroxyapatite $\left[\mathrm{Ca}_{10}\left(\mathrm{PO}_{4}\right)_{6}(\mathrm{OH})_{2}\right.$; abbreviated as $\left.\mathrm{HAp}\right]$ is an inorganic compound whose chemical composition is similar to the composition of the bone. It is a very attractive material for biomedical applications such as a bone substitute material in orthopedics and dentistry due to its excellent biocompatibility, bioactivity and osteoconduction properties (Park et al 1998a,b). Hydroxyapatite has a wider scope of applications in diverse fields like chromatography (Morales et al 2000), solid state ionics (Aizawa et al 1999, 2000), catalyst (Sugiyama et al 1999b; Tanaka et al 2000), drug delivery system (Panda et al 2001) and fuel cells (Gross et al 1998a; Verges et al 2000). It has promising application as a chemical gas sensor (Nagai et al 1988; Gross et al 1998a; Verges et al 2000).

The preparation of HAp bioceramic materials have been carried out extensively with different approaches like sol-gel (Jillavenkatesa and Condrate 1998a,b), hydrothermal processing (Yoshimura et al 1994), microwave route (Vaidhyanathan and Rao 1996), ultrasonic spray pyrolysis way (Aizawa et al 1999), precipitation route (Ikoma and

\footnotetext{
*Author for correspondence (rk2kin@yahoo.com)
}

Yamazaki 1999; Sugiyama et al 1999a), emulsion system (Furuzono et al 2001b), and sonochemical synthesis (Kim and Saito 2001). The wet chemical process, which is based on precipitation route, is the most convenient and commonly used process. This process is very simple and easy to use. The preparative reaction and the character of reaction products can be regulated (Tagai and Aoki 1980). On the other hand, use of microwaves as an alternative energy source, due to its environment-friendly, non-polluting, clean and safe approach, is also one of the most promising and excellent approaches. The great potential offered by microwave irradiation process, is the acceleration of chemical reaction (Vaidhyanathan and Rao 1996). In addition to the two above methods, hydrothermal process, which works at high temperature and high pressure, is also one of the widely used and earliest developed methods for the synthesis of hydroxyapatite. The process is not only an environmentally benign but also chemical composition and stoichiometry of the material can be controlled (Katsuki and Furuta 1999; Somiya and Roy 2000).

The dielectric behaviour of monoclinic HAp as a function of temperature, showing the phase change from monoclinic to hexagonal at a temperature of $483 \mathrm{~K}$ is already reported (Ikoma et al 1999) and the $\mathrm{CO}_{2}$ sensing behaviour of HAp has already been studied (Nagai et al 1988). 
However, the study on dielectric nature and the $\mathrm{CO}$ sensing behaviour of hexagonal HAp, is not reported.

The present paper reports the study on synthesis of hydroxyapatite by these three processes and characterization of synthetic hydroxyapatite by powder XRD for structure analysis, FTIR for identification of the functional groups, and SEM for microstructure. X-ray photoelectron spectroscopy is carried out to observe any changes in elemental composition of HAp and presence of any metallic impurities. XPS is also utilized to know the $\mathrm{Ca} / \mathrm{P}$ ratio on the HAp surface as it is one of the important parameters for HAp to be bioactive. The dielectric properties of the ceramics are also investigated as a function of frequency at room temperature. A preliminary study on gas sensing characteristic of synthetic hydroxyapatite is also reported.

\section{Experimental}

\subsection{Material synthesis}

Spec-pure grade calcium nitrate, di-ammonium hydrogen phosphate, ammonium hydroxide and calcium hydroxide were used as the starting chemicals. The stoichiometry of the calcium nitrate and di-ammonium phosphate solutions was adjusted so as to get theoretical $(\mathrm{Ca} / \mathrm{P})$ molar ratio close to $1 \cdot 66$. The precipitation was performed by slow addition of di-ammonium phosphate solution $(0.6 \mathrm{M})$ to calcium nitrate solution ( $1 \mathrm{M}$ ) under continuous and gentle stirring. The $\mathrm{pH}$ of the reaction mixture was adjusted by the addition of $\mathrm{NH}_{4} \mathrm{OH}$. As a result of reaction, milky precipitate was obtained.

In wet chemical process, the precipitate was continuously stirred for $24 \mathrm{~h}$ using magnetic stirrer. The resulting white precipitate was washed thoroughly three times with double distilled water, oven dried at about $100^{\circ} \mathrm{C}$ and crushed into powder form.

In case of hydrothermal processing, the homogeneous, milky precipitate was transferred to the stainless steel autoclave and treated hydrothermally at $100^{\circ} \mathrm{C}$ for $2 \mathrm{~h}$, $5 \mathrm{~h}$, and $8 \mathrm{~h}$ duration. The hydrothermal synthesis was also carried out at $150^{\circ} \mathrm{C}, 200^{\circ} \mathrm{C}$ temperatures for $2 \mathrm{~h}, 5 \mathrm{~h}$, and $8 \mathrm{~h}$ duration. The pressure in an autoclave was found to be changing in the range $0-14 \mathrm{~kg} / \mathrm{cm}^{2}$ depending upon the temperature of the reaction mixture. After the synthesis process, the product so obtained was washed with doubly distilled water repeatedly and dried in air oven at $100^{\circ} \mathrm{C}$. It was then crushed into powder form using agate and mortar.

For microwave processing, calcium hydroxide was taken instead of calcium nitrate whereas the other starting materials were kept the same. Since the microwave process operates at elevated temperature, use of calcium nitrate does not yield hydroxyapatite. The precipitate was obtained by adding the phosphate solution to a stirred reac- tion vessel containing calcium hydroxide solution. The $\mathrm{pH}$ of the solution was adjusted by the addition of $\mathrm{NH}_{4} \mathrm{OH}$. The precipitate was then kept inside the microwave oven (Kenstar make: Model 0M 9916E), operating at $2.45 \mathrm{GHz}$, with a variable power up to a maximum of 900 watt. The reaction mixture was exposed to microwave radiation in air for about $60 \mathrm{~min}$ at $40 \%$ power to get dry white product. The final product was scraped out of the container and crushed into powder form using agate and mortar.

Since duration of $2 \mathrm{~h}$ is sufficient for the development of HAp phase, the resulting hydroxyapatite products, prepared by three different methods, were then finally sintered at $1000^{\circ} \mathrm{C}$ for $2 \mathrm{~h}$ with PID controlled muffle furnace and allowed to cool in the furnace itself.

\subsection{Characterization}

The synthesized hydroxyapatite biomaterial ceramic powders were characterized by scanning electron microscopy, Xray diffraction, fourier transform infrared spectroscopy, and X-ray photoelectron spectroscopy. The dielectric behaviour and gas sensing character of the hydroxyapatite ceramic were also studied.

2.2a Scanning electron microscopy: The surface morphology and microstructure of the synthesized samples were observed using scanning electron microscopy (JEOL Model: JSM-5400) by coating gold on the surface to reduce charging of the samples.

2.2b X-ray diffraction: X-ray diffraction patterns were obtained with a Rigaku make X-ray diffractometer (Miniflex) with $\operatorname{CuK}_{\alpha}(\lambda=1.543 \AA)$ incident radiation. The XRD peaks were recorded in the $2 \theta$ range of $20^{\circ}-60^{\circ}$.

2.2c Fourier transform infrared spectroscopy: PerkinElmer FTIR spectrophotometer (Model System 2000) was used for identification of functional groups present in the HAp ceramic. For IR analysis, $3 \mathrm{mg}$ of the HAp powder was mixed with $300 \mathrm{mg}$ high purity $\mathrm{KBr}$. The mixture was dried at $110^{\circ} \mathrm{C}$ for $4 \mathrm{~h}$ and then compacted into a pellet form. The sample was scanned for $4000 \mathrm{~cm}^{-1}$ to $400 \mathrm{~cm}^{-1}$ with the average of 10 scans. The resolution of the spectrometer was $4 \mathrm{~cm}^{-1}$.

2.2d X-ray photoelectron spectroscopy: X-ray photoelectron spectra were recorded at room temperature by means of KRATOS AXIS-165 (dual anode) apparatus using $\mathrm{Mg} \mathrm{K} \alpha$ source having energy of $1253 \mathrm{eV}$ operated at $15 \mathrm{kV}$. All spectra were taken at the vacuum level of the order of $1 \times 10^{-9}$ mbar due to presence of $\mathrm{OH}$ group present in HAp matrix. For energy calibration, carbon $1 \mathrm{~s}$ photoelectron line (binding energy, $285 \mathrm{eV}$ ) was used. Spectra were deconvoluted using the Sun Solaris based 
Vision-2 curve resolver. The location and full width at half maximum (FWHM) for a species was first determined using the spectrum of a pure sample. The location and FWHM of products, which were not obtained as a pure species, were adjusted until the best fit was obtained. Symmetric gaussian shapes were used in all cases. Binding energies for identical samples were, in general, reproducible within $0.01 \mathrm{eV}$. All spectra were recorded in small range of binding energies so as to have a better clarity in position and shape of XPS peaks. The HAp samples were heated at elevated temperature at ambient vacuum of $1 \times 10^{-7}$ mbar in situ so as to remove impurities from the sample.

2.2e Dielectric measurements: The sintered hydroxyapatite biomaterial ceramic was compacted into a pellet of $1.0 \mathrm{~cm}$ diameter having $0.15 \mathrm{~cm}$ thickness using polyvinyl alcohol as binder material. The pellets were sintered at $1000^{\circ} \mathrm{C}$ in PID controlled air furnace for $2 \mathrm{~h}$. The flat surfaces of pellet were then coated with silver paint to form an electrode contact. These pellets were used as samples for dielectric measurements. The capacitance $(C p)$ and the quality factor $(Q)$ were recorded using a Hewlett-Packard 4284A Precision LCR meter. The measurements were carried out at room temperature, in the frequency range $20 \mathrm{~Hz}-1 \mathrm{MHz}$. The dielectric constant $\left(\varepsilon^{\prime}\right)$ was determined by the formula with a density of the order of $3 \cdot 154 \mathrm{~g} / \mathrm{cm}^{3}$ reported in literature (Slosarczyk et al 1999)

$$
\varepsilon^{\prime}=[C p \cdot t] /\left[\varepsilon_{0} \cdot A\right]
$$

where $C p$ is the capacitance of the sample, $t$ the thickness of the sample, $\varepsilon_{0}$ the permittivity of the vacuum, and $A$ the area of cross-section of the sample pellet.

2.2f Study on gas sensing characteristic: The hydroxyapatite ceramic in the form of pellets were used to carry out gas sensing studies by the method reported earlier (More et al 2003a,b). For the preparation of pellets, HAp was crushed into a fine powder in an acetone medium using agate mortar and dried under IR lamp. Then HAp was thoroughly mixed with few drops of $3 \mathrm{wt} \%$ PVA (binder). The mixture, thus formed, was used for pellet making. The powder was pressed into a pellet form under a pressure of nearly 5 tons using hydraulic press. The pellets were, then, sintered at $500^{\circ} \mathrm{C}$ for about $4-5 \mathrm{~h}$ for the removal of binder species and adsorbed gases.

These pellets were placed in a static gas characterization chamber. Initially the samples were heated slowly from room temperature to $400^{\circ} \mathrm{C}$ by varying voltage so as to avoid any thermal damage and also for the removal of moisture content. A d.c. power of $90 \mathrm{~V}$ ( $\left.V_{\text {supply }}\right)$ supplied across the pellet and voltage ( $\left.V_{\text {reference }}\right)$ across the reference resistance $(990 \mathrm{~K} \Omega)$ was noted at selected interval of temperature using digital multimeter. The resistance of the pellet, $R_{\text {sensor }}$, under study was determined by the formula

$$
R_{\text {sensor }}=\left\{\left[V_{\text {supply }}-V_{\text {reference }}\right] / V_{\text {reference }}\right\} R_{\text {reference resistance. }}
$$

To measure the change in resistance of the sensor in presence of gas, a known amount of CO gas (900 ppm) was introduced by doctor's syringe through gas inlet to the system. Again the voltage across the reference resistance was noted by reducing the temperature with the help of dimmer. The resistance of sensor pellet in presence of gas was determined using (1). The conductance of the sample in air $\left(G_{\text {air }}\right)$ and in gas atmosphere $\left(G_{\text {gas }}\right)$ was also determined (More et al 2003a,b). The sensitivity factor was calculated using the formula given as

$$
S=\left[G_{\text {gas }}-G_{\text {air }}\right] / G_{\text {air }}
$$

The variation of sensitivity factor with temperature was plotted.

\section{Results and discussion}

\subsection{Scanning electron microscopic studies}

The SEM photographs of the hydroxyapatite ceramic, shown in figure 1, depicts the overall morphology of synthesized hydroxyapatite. The examination of the micrographs revealed no distinct difference between the samples synthesized by different techniques. The microstructure of the HAp samples, as seen by scanning electron microscopy, is found to be similar to that reported in literature (Slosarczyk et al 1999; Aizawa et al 2000; Roncari et al 2000). The HAp grown at room temperature, via wet chemical route, shows non-uniform agglomerates wherein there is large variation in particle sizes (Slosarczyk and Piekarczyk 1999). Whereas, microstructure of the hydroxyapatite prepared at relatively higher temperature, via microwave irradiation way or hydrothermal route, shows uniform grain growth along with high porosity.

\subsection{X-ray diffraction studies}

Figure 2 displays the intensity of diffracted X-rays from various planes as a function of $2 \theta$ value for the HAp biomaterial ceramic prepared by wet chemical process, microwave irradiation and hydrothermal routes, respectively. The XRD phase identification was performed using JCPDS standard XRD card (09-432) for hydroxyapatite, wherein the $d$ values are found to be in good agreement with the standard data. The presence of HAp characteristic diffraction peaks, along with the additional HAp peaks near $30.6^{\circ}, 33^{\circ}, 38.8^{\circ}, 45 \cdot 6^{\circ}, 48 \cdot 4^{\circ}, 52 \cdot 2^{\circ}$ confirms that the synthesized material is HAp. The additional XRD peaks appearing at $26.8^{\circ}$ and $43.5^{\circ}$, in 

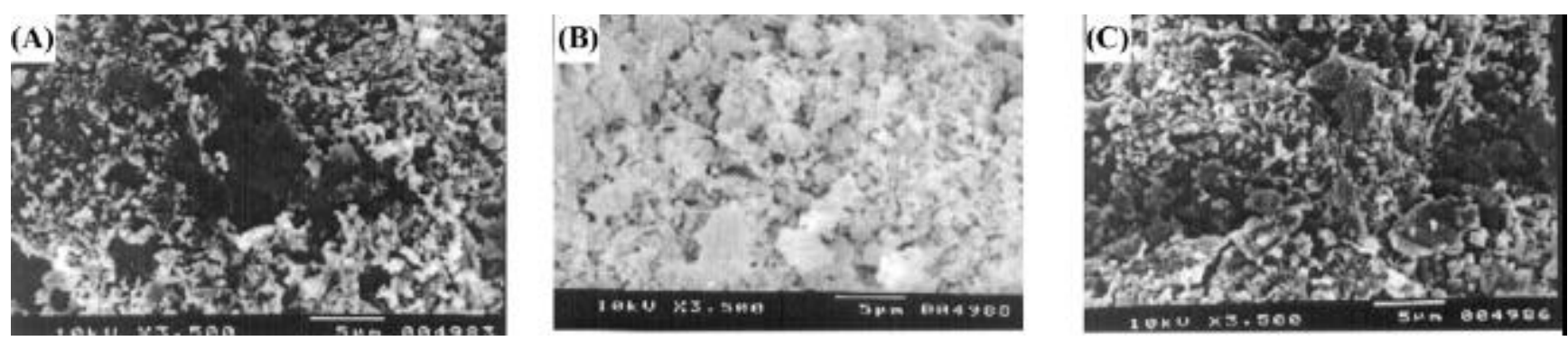

Figure 1. Scanning electron micrographs for sintered hydroxyapatite powder synthesized by (A) wet chemical process, (B) microwave irradiation method and $(\mathbf{C})$ hydrothermal method.
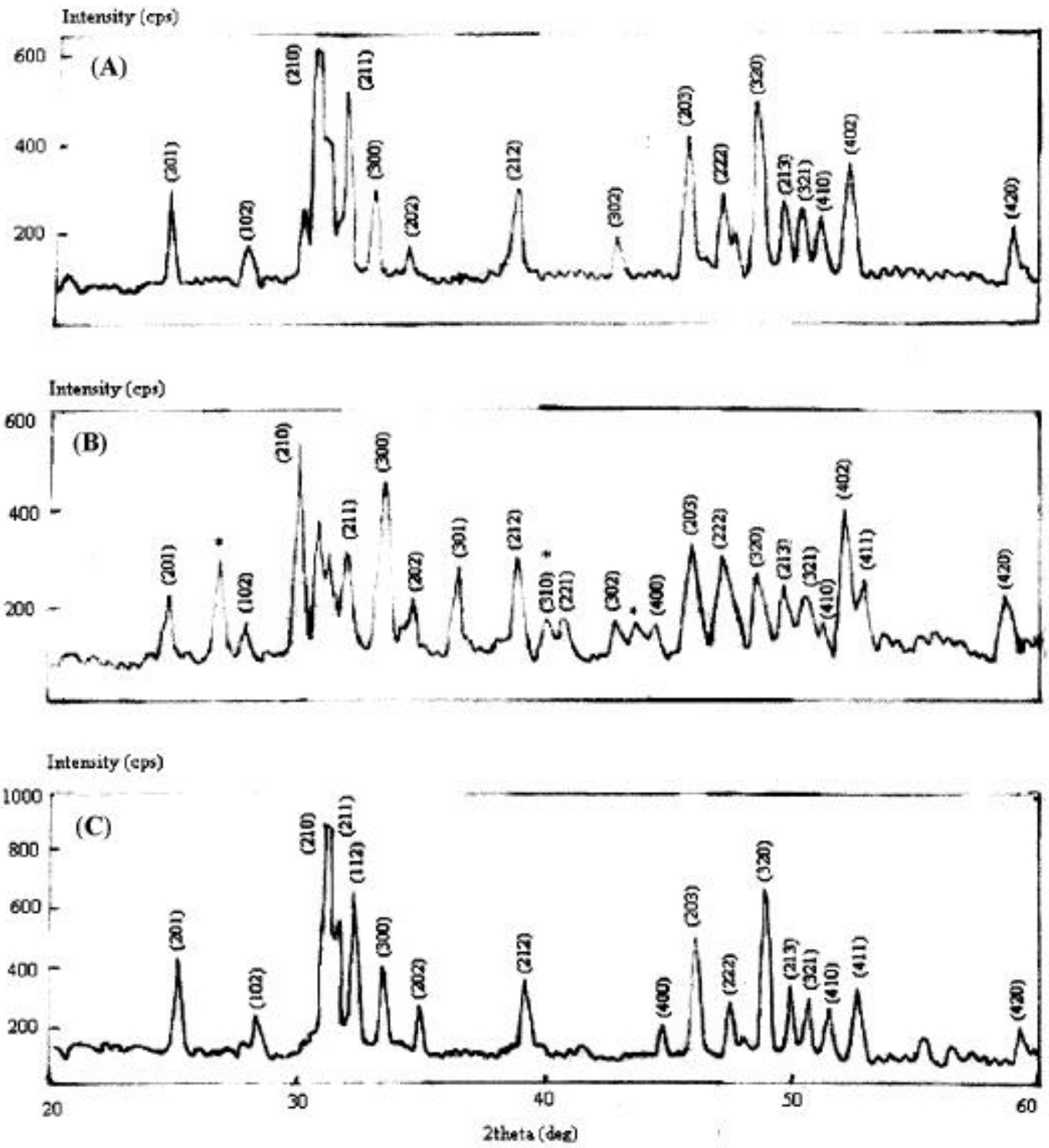

Figure 2. Typical powder X-ray diffraction pattern of sintered hydroxyapatite synthesized by (A) wet chemical process, $(\mathbf{B})$ microwave irradiation method and $(\mathbf{C})$ hydrothermal method. 
Table 1. The average crystallite size calculated using Scherrer's formula for the samples prepared by three different techniques.

\begin{tabular}{lll}
\hline $\begin{array}{l}\text { Technique used for } \\
\text { synthesis }\end{array}$ & \multicolumn{1}{c}{ Sample details } & $\begin{array}{c}\text { Average crystallite size } \\
(\mu \mathrm{m})\end{array}$ \\
\hline Wet chemical process & Sample synthesized at room temperature & $0 \cdot 032$ \\
Microwave process & Synthesized at $2 \cdot 45 \mathrm{GHz}$ & $0 \cdot 054$ \\
Hydrothermal process & Samples synthesized at $200^{\circ} \mathrm{C}$ & $0 \cdot 045$ \\
& Samples synthesized at $100^{\circ} \mathrm{C}$ & $0 \cdot 031$ \\
\hline
\end{tabular}

microwave processed HAp, are attributed to trace of carbonate impurities present in starting material and are in conformation with FTIR data. Moreover, the peak at $40^{\circ}$ corresponds to HAp reflection corresponding to (310) plane. According to literature, the calcium carbonate peak appears at $39.9^{\circ}$. However, it is very difficult to assign this peak to impurity because of overlapping of carbonate and HAp planes. However, each profile matches well with the standard data and also with that reported in the literature (Shaoxian et al 1993; Vaidhyanathan and Rao 1996; Katsuki and Furuta 1999). Hence, it can be concluded that grown HAp favours hexagonal phase. The appearance of sharp peaks shows high degree of crystallinity of the samples and show no other additional phases. The crystallite size was estimated from line broadening of the diffraction lines using Scherrer's equation

$$
D=[k \cdot \lambda] /[\beta \cdot \cos \theta]
$$

where $\beta$ is the full width at half maxima of the powder diffraction peak in radians, $\theta$ the reflection angle of the peak, $k$ a constant nearly equal to 0.9 and $\lambda$ the wavelength of the X-ray radiation.

The prime reflections with considerable intensities such as (201), (210), (211), (300), (212), (203) and (320) are employed for calculation of Scherrers broadening. The average crystallite size, calculated using Scherrer's formula, is reported in table 1.

\subsection{Fourier transform infrared spectroscopic analysis}

The FTIR spectra of HAp samples, synthesized by wet chemical process, microwave irradiation technique and hydrothermal process, are shown in figure 3 . The IR spectra of the biomaterial show the absorption bands at $3572 \mathrm{~cm}^{-1}$ (Furuzono et al 2001a,b) and $3433 \mathrm{~cm}^{-1}$ which correspond to stretching mode of hydroxyl group (Bertoni et al 1998; Tampieri et al 2000). The hydroxyl libration mode is found to be present near $632 \mathrm{~cm}^{-1}$ (Gross et al 1998b; Ikoma et al 1999). The band due to $v_{3}$ vibrations of hydroxyl group, observed for HAp synthesized by microwave route, is present at $1625 \mathrm{~cm}^{-1}$ (Komath et al 2000), whereas the bands at 1636 for other samples are due to $\left(\mathrm{NO}_{3}\right)$ group (Aizawa et al 1999). The absorption bands appearing at $418 \mathrm{~cm}^{-1}$ (Furuzono et al 2001a),
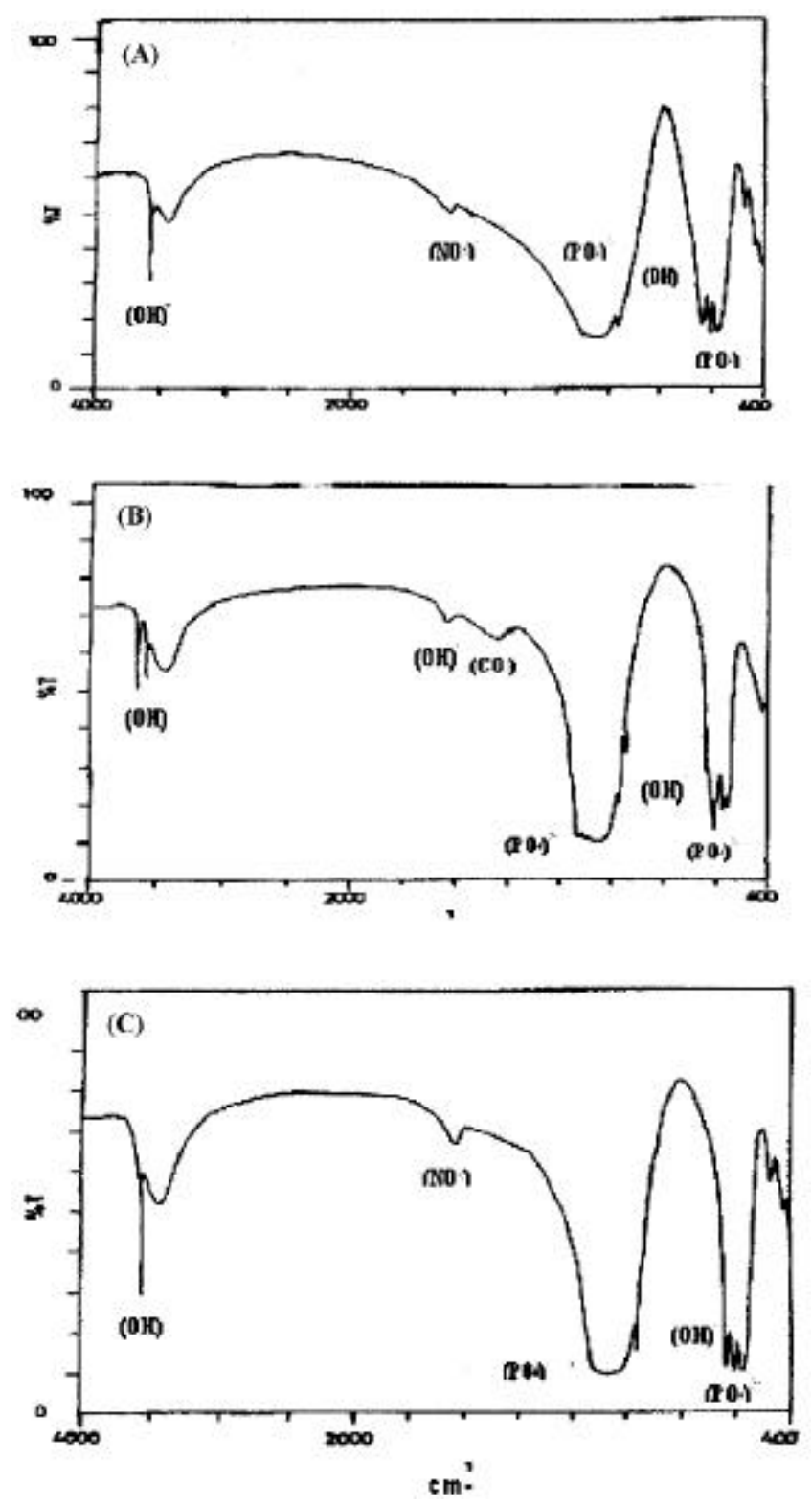

Figure 3. Infrared absorption spectra of HAp, after heat treatment at $1000^{\circ} \mathrm{C}$, synthesized by (A) wet chemical process, (B) microwave irradiation method and (C) hydrothermal method.

$575 \mathrm{~cm}^{-1}$ (Jillavenkatesa et al 1999) and $601 \mathrm{~cm}^{-1}$ can be attributed to the $v_{4}$ fundamental bending mode of $\left(\mathrm{PO}_{4}^{3-}\right)$ functional group (Park et al 1998a; Rivera et al 1999) 
and the peaks in the range $1089-1039 \mathrm{~cm}^{-1}$ are due to $v_{3}$ vibrations of $\left(\mathrm{PO}_{4}^{3-}\right)$ (Jillavenkatesa and Condrate 1998a; Komath et al 2000). The bands at $974 \mathrm{~cm}^{-1}$ and $963 \mathrm{~cm}^{-1}$ are due to $v_{1}$ fundamental mode of $\left(\mathrm{PO}_{4}^{3-}\right)$ (Furuzono 2001a,b). The IR spectrum of the biomaterial synthesized by microwave technique shows additional absorption band at $3644 \mathrm{~cm}^{-1}$ (Ishikawa et al 2000) which corresponds to hydroxyl stretching mode associated with surface $\mathrm{P}-\mathrm{OH}$ groups and presence of small amount of $\mathrm{CO}_{3}$ can be evidenced by the bands appearing at $1459 \mathrm{~cm}^{-1}$ showing the formation of carbonate apatite (Tampieri et al 1997). This is attributed to the trace impurities present in the starting material.

\subsection{XPS studies}

By looking at general scan up to $1000 \mathrm{eV}$, traces of metallic and other impurities were not seen, indicating the HAp sample to be pure and clean in nature. The major peaks, found in the XPS spectra, are in agreement with the reported literature (Raikar et al 1996; Yoshida et al 2000) and were attributed to chemical state of $\mathrm{Ca}, \mathrm{P}$, and $\mathrm{O}$ elements in Ca-HAp matrix (Panda et al 2001; Baillez et al 2004). Figure 4 displays typical XPS scan for $\mathrm{Ca}, \mathrm{P}$, and $\mathrm{O}$ atoms for HAp synthesized by three different processing routes. The peak positions of $\mathrm{Ca}_{3 / 2}, \mathrm{P}_{1 / 2}, \mathrm{O}_{1 / 2}$ in terms of binding energy for the samples prepared by
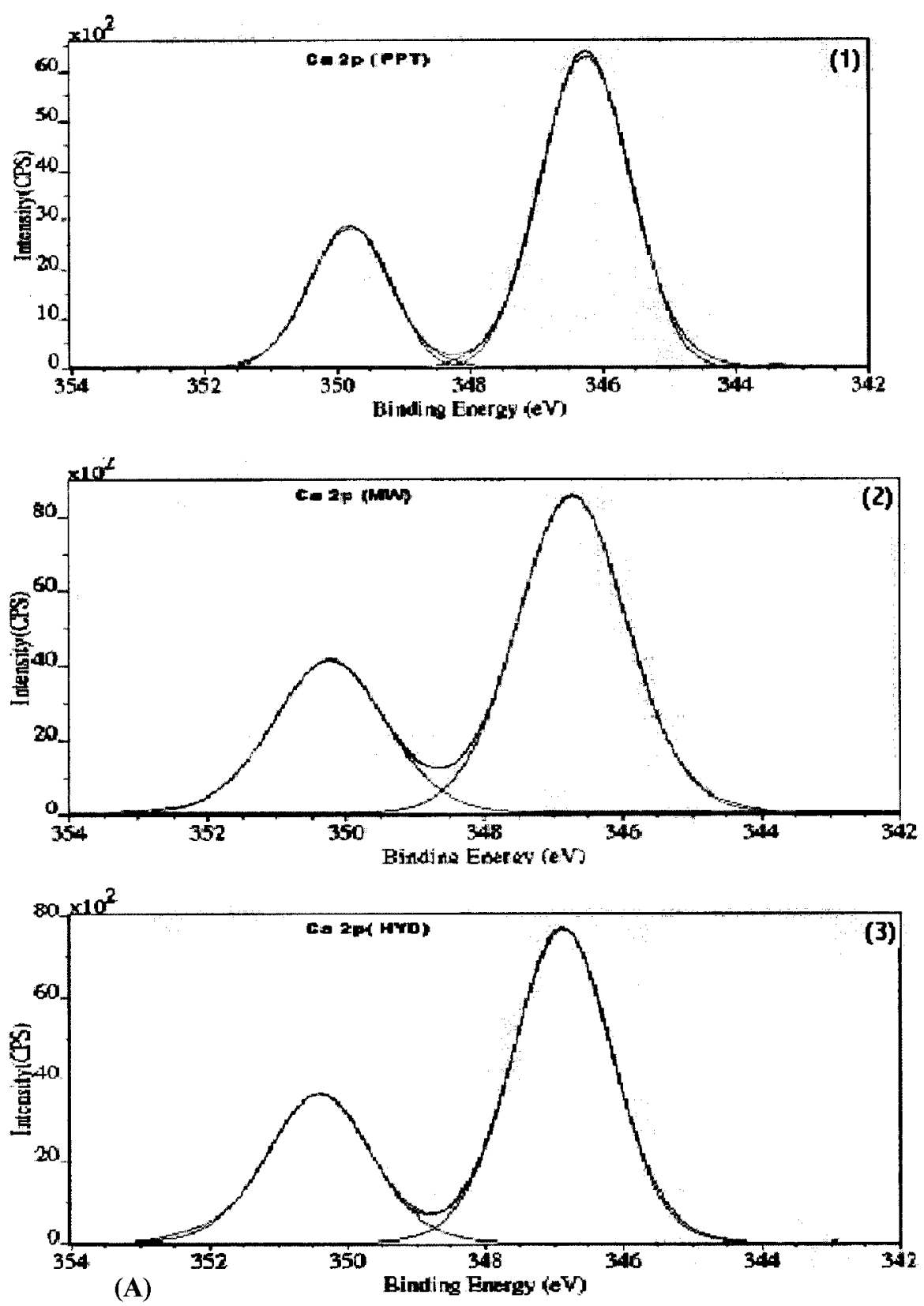

Figure 4(A). For caption see page no. 542. 
three different routes are almost identical, which clearly reveals that these elements are in similar environment in these samples. However, peak shapes are found to change indicating the variation in atomic percentage in the HAp matrix prepared by these three techniques. The observed peak positions near $346 \mathrm{eV}$ and $350 \mathrm{eV}$ are attributed to $\mathrm{Ca} 2 p_{3 / 2}$ and $\mathrm{Ca} 2 p_{1 / 2}$, respectively. The $\mathrm{O} 1 s$ peaks near $530 \mathrm{eV}$ and $532 \mathrm{eV}$ are due to oxygen associated with phosphate group in HAp and adsorbed water, respectively. Table 2 summarizes various parameters of HAp as deduced from XPS studies. Quantifying the elements observed in the survey scans, $\mathrm{Ca} / \mathrm{P}$ ratio has been determined by taking the area under the characteristic peaks of Ca $2 p$ and $\mathrm{P} 2 p$. HAp prepared by microwave irradiation technique is found to be rich in calcium whereas the one synthesized by hydrothermal method is deficient in calcium. HAp, prepared by chemical method, exhibited the elemental ratio $\mathrm{Ca} / \mathrm{P}$ to be approximately 1.63 .

\subsection{Dielectric measurements}

The typical variation of dielectric constant $\left(\varepsilon^{\prime}\right)$ of the HAp sample with frequency of the applied field is represented in figure 5A. The dielectric constant for all the samples is found to be at $400 \mathrm{~Hz}$, in the range 9-13. It is reported in the literature that the dielectric constant of HAp at $291.5 \mathrm{~K}$ was 15.4 at $100 \mathrm{~Hz}$ (Ikoma et al 1999). The difference in the values can be attributed to the difference in the processing and structure of the end pro-
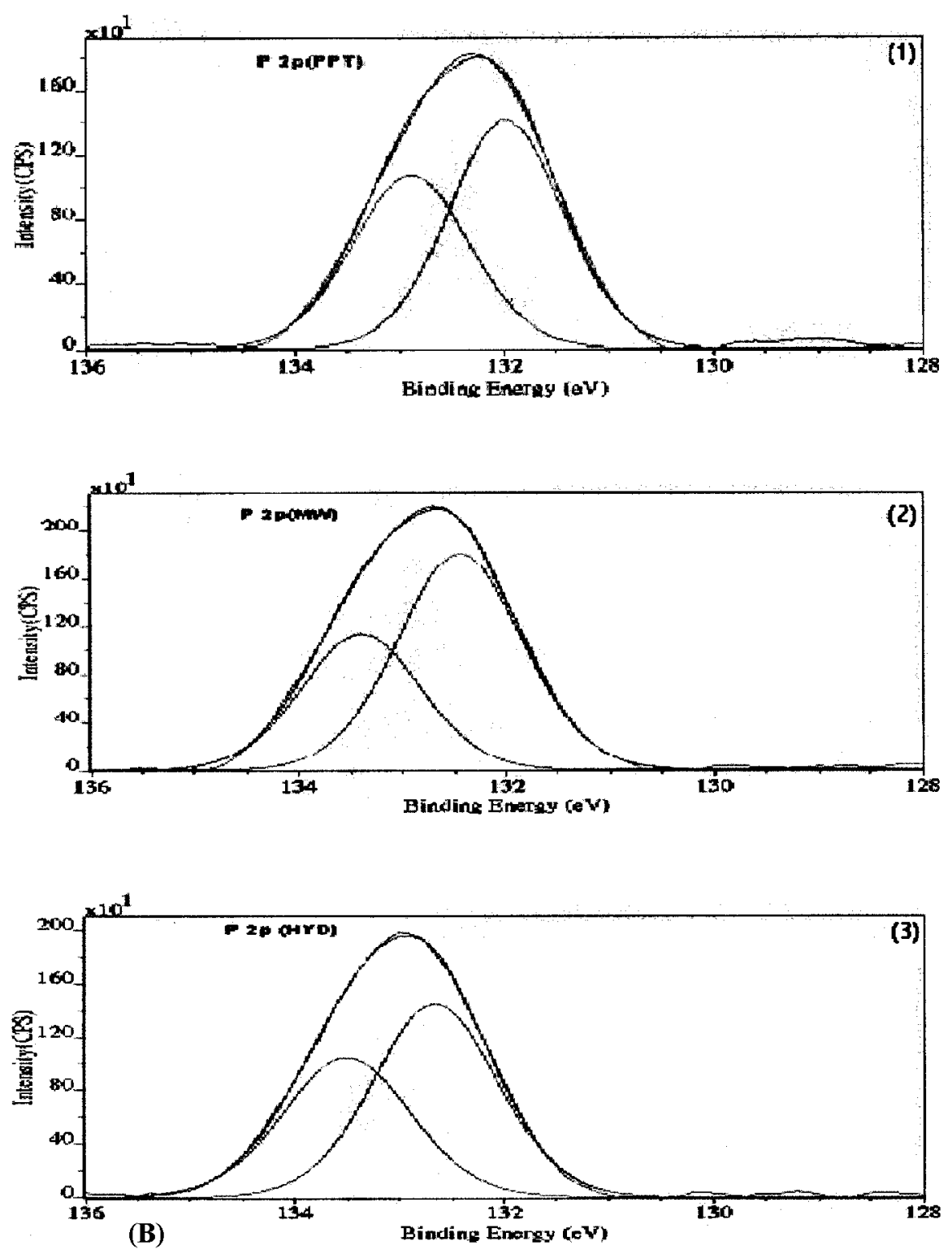

Figure 4(B). For caption see page no. 542. 
duct. It is observed that $\varepsilon^{\prime}$ decreases continuously with increase in applied frequency and then reaches a constant value for all the samples. Dielectric loss $\left(\varepsilon^{\prime \prime}\right)$ of the HAp sample also shows the dielectric dispersion, as shown in figure 5B. Figure 5C displays the variation of $Q$ value as a function of frequency. It is observed that $Q$ value increases linearly with frequency showing a small peak near $400 \mathrm{KHz}$. The hydroxyapatite, prepared by other routes, also shows the same trend.
In normal dielectric behaviour, the dielectric constant decreases with increasing frequency reaching a constant value, depending on the fact that beyond certain frequency of the electric field, the dipoles do not follow the alternating field. In ionic crystals, the total polarization is electronic and ionic in nature. The hydroxyapatite crystal structure is also ionic in nature. It consists of $\mathrm{OH}^{-}$ions aligned in columns along the $c$-axis of the crystal along with $\mathrm{Ca}^{2+}$ and $\left(\mathrm{PO}_{4}^{3-}\right)$ ions. Since the hydroxyapatite
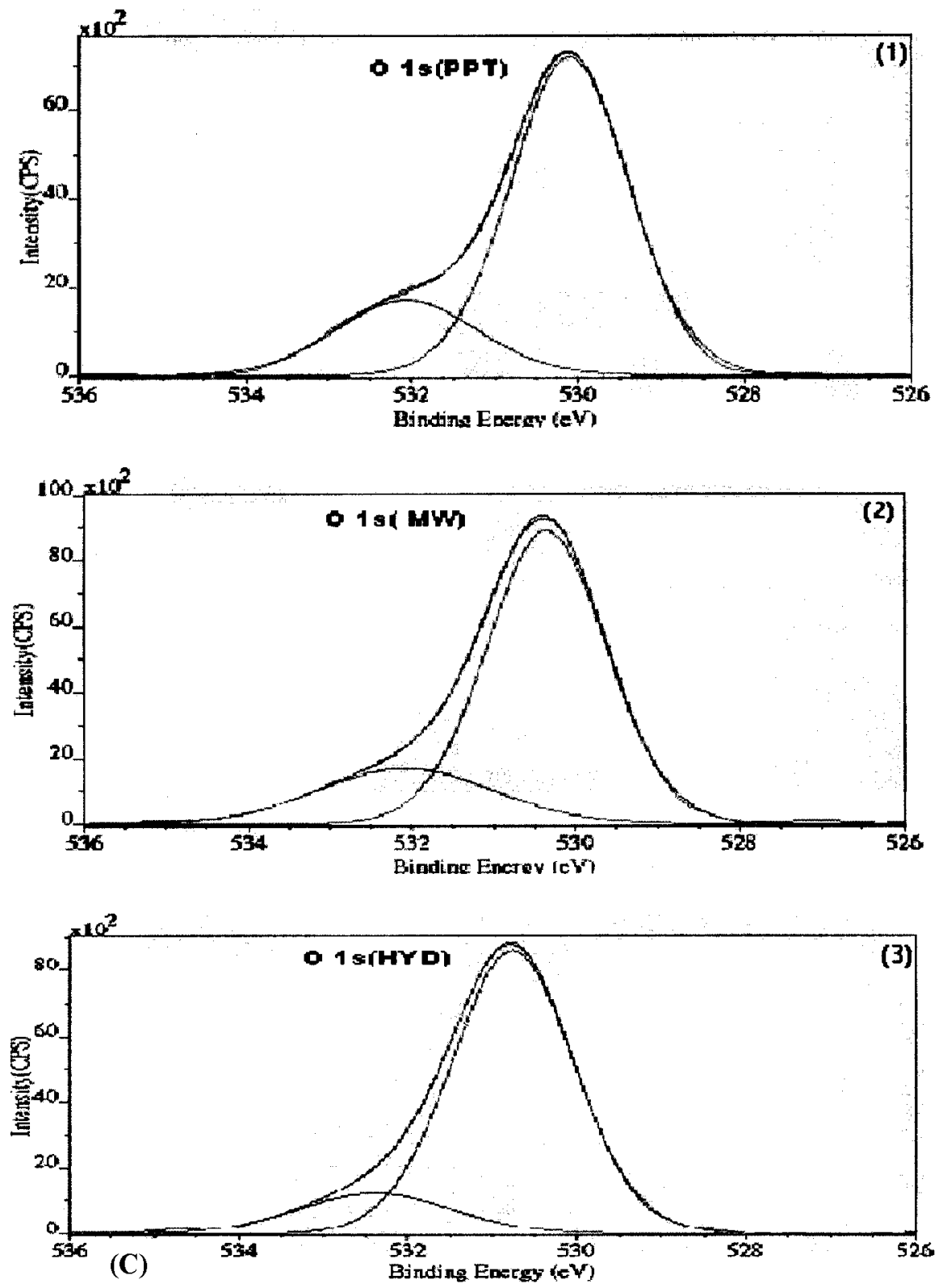

Figure 4. (A) XPS scan of Ca $2 p$ peak for HAp synthesized by (1) chemical method, (2) microwave irradiation and (3) hydrothermal method, (B) XPS scan of P $2 p$ peak for HAp synthesized by (1) chemical method, (2) microwave irradiation and (3) hydrothermal method and (C) XPS scan of O $1 s$ peak for HAp synthesized by (1) chemical method, (2) microwave irradiation and (3) hydrothermal method. 
Table 2. Peak positions and associated chemical states as deduced from XPS spectra.

\begin{tabular}{|c|c|c|c|c|}
\hline $\begin{array}{l}\text { Sample preparation } \\
\text { process }\end{array}$ & Level & Chemical states & $\begin{array}{l}\text { Binding energy } \\
(\mathrm{eV})\end{array}$ & Peak area \\
\hline Wet chemical process & $\begin{array}{l}\mathrm{Ca} 2 p_{1 / 2} \\
\mathrm{Ca} 2 p_{3 / 2} \\
\mathrm{P} 2 p \\
\mathrm{O} 1 s\end{array}$ & $\begin{array}{l}\mathrm{Ca}^{2+} \\
\mathrm{Ca}^{2+} \\
\left(\mathrm{PO}_{4}\right)^{3-} \\
\mathrm{O}^{2-\delta} \\
\mathrm{O}^{2}\end{array}$ & $\begin{array}{l}349 \cdot 812 \\
346 \cdot 232 \\
132 \cdot 897 \\
131 \cdot 929 \\
532 \cdot 078 \\
530 \cdot 108\end{array}$ & $\begin{array}{l}28 \cdot 6 \\
71 \cdot 4 \\
43 \cdot 8 \\
56 \cdot 2 \\
22 \cdot 2 \\
77 \cdot 8\end{array}$ \\
\hline Microwave irradiation process & $\begin{array}{l}\text { Ca } 2 p_{1 / 2} \\
\text { Ca } 2 p_{3 / 2} \\
\text { P } 2 p \\
\text { O1s }\end{array}$ & $\begin{array}{l}\mathrm{Ca}^{2+} \\
\mathrm{Ca}^{2+} \\
\left(\mathrm{PO}_{4}\right)^{3-} \\
\mathrm{O}^{2-\delta} \\
\mathrm{O}^{2}\end{array}$ & $\begin{array}{l}350 \cdot 243 \\
346 \cdot 724 \\
133 \cdot 416 \\
132 \cdot 450 \\
532 \cdot 105 \\
530 \cdot 361\end{array}$ & $\begin{array}{l}33 \cdot 1 \\
66 \cdot 9 \\
37 \cdot 6 \\
62 \cdot 4 \\
22 \\
78\end{array}$ \\
\hline Hydrothermal process & $\begin{array}{l}\text { Ca } 2 p_{1 / 2} \\
\text { Ca } 2 p_{3 / 2} \\
\text { P } 2 p\end{array}$ & $\begin{array}{l}\mathrm{Ca}^{2+} \\
\mathrm{Ca}^{2+} \\
\left(\mathrm{PO}_{4}\right)^{3-} \\
\mathrm{O}^{2-\delta} \\
\mathrm{O}^{2}\end{array}$ & $\begin{array}{l}350 \cdot 432 \\
346 \cdot 871 \\
133 \cdot 509 \\
132 \cdot 641 \\
532 \cdot 420 \\
530 \cdot 765\end{array}$ & $\begin{array}{l}33 \\
67 \\
42 \cdot 6 \\
87 \cdot 4 \\
15 \cdot 1 \\
84 \cdot 9\end{array}$ \\
\hline
\end{tabular}

structure contains several ions, which move around in the lattice, the mechanism of dipole alteration with frequency of applied field becomes very complex. But, it is the behaviour of dipole moment of the hydroxyl ions, the major contribution of which, is responsible for the dielectric nature of hydroxyapatite. At low frequencies, polarization follows the alterations of the field without any significant lag but at high frequencies the relatively heavy positive and negative ions cannot follow the field variations. As frequency increases, a particular frequency is reached where, the dipole moment of the hydroxyl ions cannot sustain with the field variation and hence polarization decreases, which results in the decrease of $\varepsilon^{\prime}$.

\subsection{Gas sensing behaviour}

The typical variation of sensitivity factor in $\mathrm{CO}$ gas atmosphere as function of temperature for the hydroxyapatite synthesized by microwave irradiation process is presented in figure 6. The response shows that hydroxyapatite is sensitive to $\mathrm{CO}$ at temperature near to $125^{\circ} \mathrm{C}$. This shows that it has specific temperature sensitivity for $\mathrm{CO}$ since at all other temperatures the sensitivity response of $\mathrm{CO}$ is meagre. Also, the variation in sensitivity factor near $125^{\circ} \mathrm{C}$ is found to be very sharp. This confirms that hydroxyapatite is a very good $\mathrm{CO}$ sensor at the temperature near to $125^{\circ} \mathrm{C}$. The sensitivity factor, as large as $0 \cdot 8$, was observed for $\mathrm{CO}$ gas having $900 \mathrm{ppm}$ concentration. The gas sensing mechanism is due to adsorption and desorption phenomena. The hydroxyapatite pellet surface possesses $\mathrm{P}-\mathrm{OH}$ groups which act as adsorption sites for the gas to be sensed. Thus, there is wider distribution of sites available on the surface of HAp. Moreover, the hydroxyapatite structure is porous. These pores also work as conduction paths for adsorbed gas molecules. At room temperature the rate of adsorption is generally found to be higher than that of desorption. With the increase in temperature of the sample pellet, the rate of adsorption decreases and at a particular temperature the rate of adsorption and desorption becomes equal. At this temperature, sensitivity factor increases sharply, indicating the optimum temperature at which the material can be used as a sensor. Similar results have been obtained for the samples prepared by other two techniques, wherein the optimum temperature is found to be near $125^{\circ} \mathrm{C}$. The $\mathrm{CO}$ sensor working at $150^{\circ} \mathrm{C}$ has also been reported (More et al 2003a,b). The present sensor is working at still lower temperature. The possible mechanism for getting the material reduced could be as follows:

$$
\begin{aligned}
& \text { OH group }+\mathrm{CO} \rightarrow \mathrm{CO}_{2}+\mathrm{H}_{2} \text { or } \mathrm{OH} \text { group }+ \\
& \mathrm{CO} \rightarrow \mathrm{CO}_{2}+\mathrm{H}_{2} \mathrm{O} .
\end{aligned}
$$

As the gas is getting oxidized, the conductivity of the material increases.

\section{Conclusions}

Microwave and hydrothermal routes yield uniform grain growth along with highly porous crystalline HAp material. The microwave irradiation process requires less time for the synthesis of hydroxyapatite compared to other two processes. The formation of hexagonal hydroxyapatite 

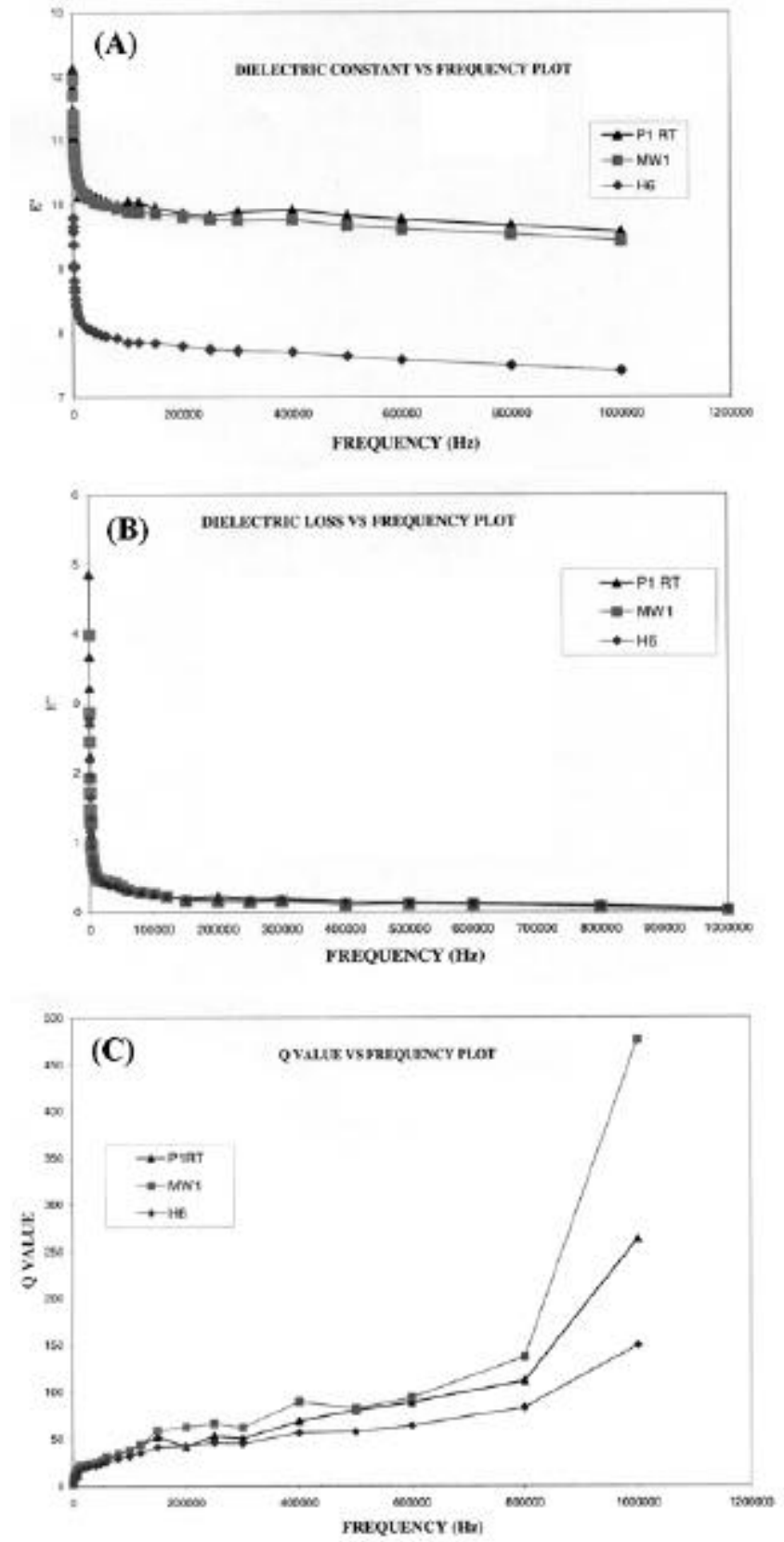

Figure 5. (A) Typical variation of dielectric constant $\left(\varepsilon^{\prime}\right)$ of sintered HAp biomaterial with frequency of the applied field at room temperature: $(\boldsymbol{\Delta})$, wet chemical method; $(\mathbf{\square})$, microwave irradiation method and ( $\bullet$ ), hydrothermal method, (B) dielectric loss $\left(\varepsilon^{\prime \prime}\right)$ of HAp biomaterial ceramic with frequency of the applied field at room temperature $(\boldsymbol{\Lambda}$, wet chemical method, $\boldsymbol{\square}$, microwave irradiation method, $\bullet$, hydrothermal method) and (C) variation of quality factor of HAp biomaterial as a function of frequency of the applied field at room temperature $(\boldsymbol{\Lambda}$, wet chemical method, $\mathbf{\square}$, microwave irradiation method and $\bullet$, hydrothermal method).

phase is identified by XRD. The presence of predominant characteristic FTIR peaks corresponding to the $\left(\mathrm{PO}_{4}^{3-}\right)$, $(\mathrm{OH})^{-}$and $\left(\mathrm{NO}_{3}\right)^{-}$groups indicates the development of HAp phase. The grain size is found to be in the range $31-$

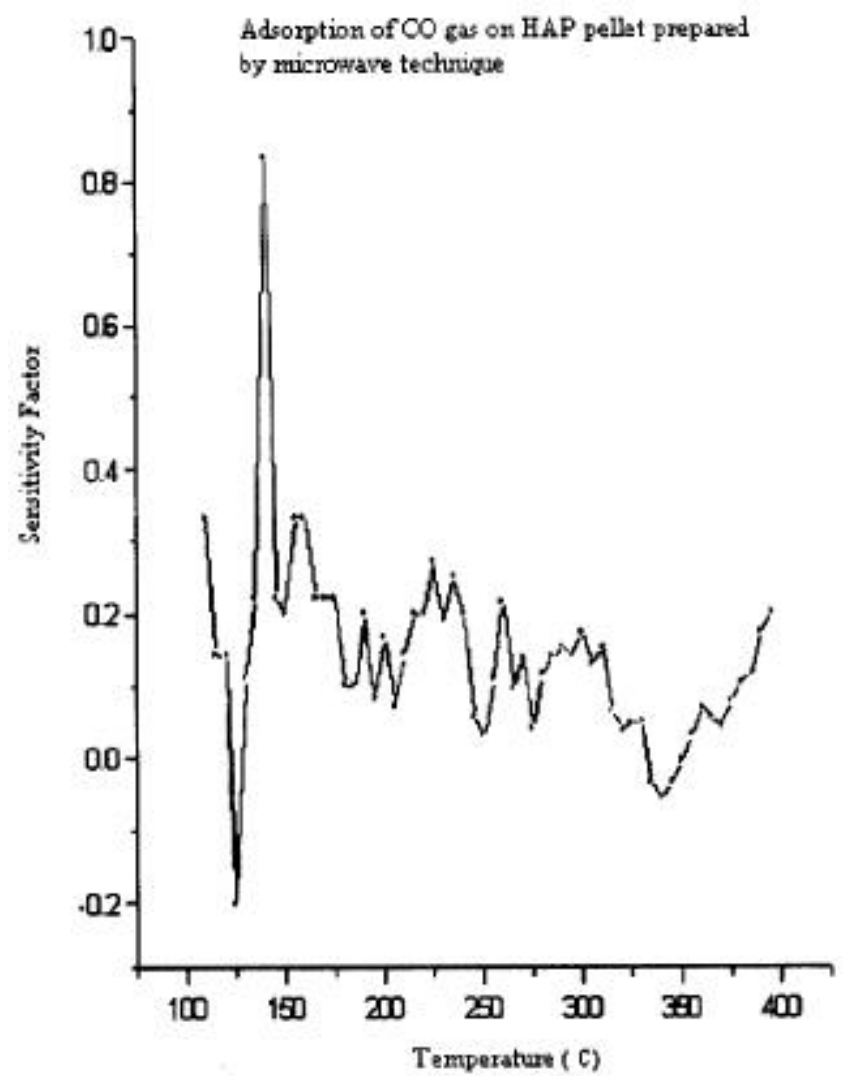

Figure 6. Variation of sensitivity factor as a function of temperature of the hydroxyapatite sensor pellet synthesized by microwave irradiation method.

$54 \mathrm{~nm}$. The dielectric constant is in the range 9-13. Hydroxyapatite seems to be a potential candidate to act as $\mathrm{CO}$ sensor at an optimum temperature near $125^{\circ} \mathrm{C}$. The $\mathrm{Ca} / \mathrm{P}$ ratio is in the range of $1.6-1.7$ as seen by XPS studies, a property which is important in biomedical applications.

\section{Acknowledgements}

Thanks are due to Hon'ble Vice-Chancellor, Dr D B Yedekar, SRTM University, Nanded, for his valuable guidance and constant encouragement. The kind help provided by Prof. B K Chougule, Department of Physics, Shivaji University, Kolhapur, in dielectric measurements and Prof. P P Patil, School of Physical Sciences, Uttar Maharashtra University, Jalgaon, for XRD data, is gratefully acknowledged.

\section{References}

Aizawa M, Hanazawa T, Itatani K, Howell F S and Kishioka A 1999 J. Mater. Sci. 342865

Aizawa M, Howell F S, Itatani K, Yokogawa Y, Nishizawa K, Toriyama M and Kameyama T 2000 J. Ceram. Soc. Jpn 108 249 
Bertoni E, Bigi A, Cajazzi G, Gandolfi M, Panzavolta S and Roveri N 1998 J. Inorg. Biochem. 7229

Baillez S, Nzihou A, Beche E and Flamant G 2004 Proc. Safety and Env. Protect. 82175

Furuzono T, Sonoda K and Tanaka J 2001a J. Biomed. Mater. Res. 569

Furuzono T, Walsh D, Sato K, Sonoda K and Tanaka J 2001b J. Mater. Sci. Letts 20111

Gross K A, Berndt C C, Stephens P and Dinnebier R 1998a J. Mater. Sci. 333985

Gross K A, Gross V and Berndt C C 1998b J. Am. Ceram. Soc. 81106

Ikoma T and Yamazaki A 1999 J. Solid State Chem. 144272

Ikoma T, Yamazaki A, Nakamura S and Akao M 1999 J. Mater. Sci. Letts 181225

Ishikawa T, Teramachi A, Tanaka H, Yasukawa A and Kandori K 2000 Langmuir 1610221

Jillavenkatesa A and Condrate Sr R A 1998a J. Mater. Sci. 33 4111

Jillavenkatesa A and Condrate Sr R A 1998b Canadian J. Anal. Sci. \& Spectrosc. $\mathbf{4 3} 161$

Jillavenkatesa A, Hoelzer D T and Condrate Sr R A $1999 \mathrm{~J}$. Mater. Sci. 344821

Katsuki H and Furuta S 1999 J. Am. Ceram. Soc. 822257

Kim W and Saito F 2001 Ultrason. Sonochem. 885

Komath M, Verma H K and Sivakumar R 2000 Bull. Mater. Sci. 23135

Morales J G, Burgues J J, Boix T, Fraile J and Clemente R R 2000 Cryst. Res. Technol. 3615

More P S, Khollam Y B, Deshpande S B, Date S K, Karekar R N and Aiyer R C 2003a Mater. Letts 572177

More P S, Khollam Y B, Deshpande S B, Date S K, Karekar R N and Aiyer R C 2003b Mater. Letts 58205

Nagai M, Nishino T and Saeki T 1988 Sensors \& Actuators 15145

Panda R N, Ming-Fa H, Chung R J and Chin T S 2001 Jpn J. Appl. Phys. 405030
Park E, Condrate Sr R A and Lee D 1998a Mater. Letts 3638

Park E, Condrate Sr R A and Hoelzer D T 1998b J. Mater. Sci.: Mater. Med. 9643

Raikar G N, Ong J L and Lucas L C 1996 Surf. Sci. Spectra 49

Rivera E M, Araiza M, Brostow W, Castano V M, Diaz-Estrada J R, Hernandez R and Rodriguez J R 1999 Mater. Letts 41 128

Roncari E C, Galassi C and Pinasco P 2000 J. Mater. Sci. Letts 1933

Shaoxian Z, Zhixiong Y, Ping L, Guanghong X and Wanpeng C 1993 Proc. Mater. Res. Soc. Symp. 292271

Slosarczyk A and Piekarczyk J 1999 Ceram. Int. 25561

Slosarczyk A, Stobierska E and Paszkiewicz Z 1999 J. Mater. Sci. Letts 181163

Somiya S and Roy R 2000 Bull. Mater. Sci. 23453

Sugiyama S, Yasutomi T, Moriga T, Hayashi H and Moffat J B 1999a J. Solid State Chem. 142319

Sugiyama S, Nakanishi T, Ishimura T, Moriga T, Hayashi H, Shigemoto N and Moffat J B 1999b J. Solid State Chem. 143 296

Tagai H and Aoki H 1980 in Mechanical properties of biomaterials (eds) G W Hasting and D F Williams (New York: John Wiley and Sons Ltd.) pp 477-488

Tampieri A, Celotti G, Szontagh F and Landi E 1997 J. Mater. Sci.: Mater. Med. 829

Tampieri A, Celotti G, Suprio S and Mingazzini M 2000 Mater. Chem. and Phys. 6454

Tanaka H, Chikazawa M, Kandori K and Ishikawa T 2000 Phys. Chem. Chem. Phys. 2647

Vaidhyanathan B and Rao K J 1996 Bull. Mater. Sci. 191163

Verges M A, Ganzalez C F, Gallego M M, Solier J D, Cachadina I and Matijevic E 2000 J. Mater. Res. 152526

Yoshimura M, Suda H, Okamoto K and Ioku K 1994 J. Mater. Sci. 293399

Yoshida Y, Nakayama Y, Snauwaert J, Hellemans L, Lambrechts P, Vanherle G and Wakasa K 2000 J. Dental Res. 79709 